Service social

\title{
Les pratiques évaluatives : enjeux, stratégies et principes
}

\section{André Beaudoin, Richard Lefrançois et Francine Ouellet}

Volume 35, numéro 1-2, 1986

Recherche - Action - Évaluation

URI : https://id.erudit.org/iderudit/706301ar

DOI : https://doi.org/10.7202/706301ar

Aller au sommaire du numéro

Éditeur(s)

École de service social de l'Université Laval

ISSN

1708-1734 (numérique)

Découvrir la revue

Citer cet article

Beaudoin, A., Lefrançois, R. \& Ouellet, F. (1986). Les pratiques évaluatives :

enjeux, stratégies et principes. Service social, 35(1-2), 188-213.

https://doi.org/10.7202/706301ar d'utilisation que vous pouvez consulter en ligne.

https://apropos.erudit.org/fr/usagers/politique-dutilisation/ 
BEAUDOIN, André, professeur à l'École de service social de l'Université Laval.

LEFRANÇOIS, Richard, professeur au Département de service social de l'Université de Sherbrooke.

OUELLET, Francine, professeur à l'École de service social de l'Université Laval.

\section{Les pratiques évaluatives: enjeux, stratégies et principes ${ }^{1}$}

\section{André Beaudoin Richard Lefrançois Francine Ouellet}

Avant tout, cet article se veut une conclusion à l'ensemble des thèmes qui ont été traités ici par les différents auteurs; c'est aussi une relance par rapport au questionnement actuel sur l'utilisation de la recherche, ce qui suppose une compréhension des transformations qui marquent actuellement les politiques sociales, les pratiques d'intervention et la recherche.

Les mutations qui s'observent dans le rôle et les engagements sociaux de l'État, l'incertitude régnant dans les milieux de pratique suite aux récentes restructurations et, comme résultante, la crise que traverse l'intervention, s'accompagnent d'impulsions nouvelles caractérisant la recherche sociale. En effet, les nécessités nouvelles de l'intervention et de la décision politico-administrative orientent les pratiques de recherche de façon significative et concrète: l'emphase étant dorénavant l'action et l'évaluation. (Lefrançois et Soulet, 1983).

Les apports du présent numéro témoignent manifestement de l'existence d'une double orientation marquant la recherche sociale appliquée : d'une part, celle-ci est appelée à s'associer au processus de validation des pratiques institutionnelles et, d'autre part, à réarticuler ou réinventer ses rapports avec le terrain.

Dans l'esprit du gestionnaire, le recours notamment aux pratiques évaluatives s'impose doublement: 1) dans la perspective traditionnelle de distribution des services, elles visent à optimiser le rendement et 
l'efficacité des programmes existants et, en outre, à exercer une surveillance plus étroite de la pratique professionnelle; 2) dans l'optique des orientations alternatives au modèle institutionnel et professionnel, elles représentent l'espoir d'apporter la preuve de la viabilité des programmes préventifs et des approches nouvelles en intervention (services de maintien à domicile, programmes sur la qualité de vie, renforcement des réseaux naturels de support, etc.).

Par ailleurs, la ferveur actuelle des expériences de rechercheaction traduit sans doute cette même nécessité de réaffirmer le sens et l'efficacité des pratiques dans un effort de domestication du savoir via la participation accrue des agents ou groupes impliqués. À cet égard, une analyse de la littérature récente sur l'évaluation révèle que la "subjectivité des publics" constitue un matériau, une source d'information importante pour juger des résultats d'un programme ou d'une intervention (Von Schoenberg, 1985). Il n'y a donc rien d'étonnant à ce que des stratégies de recherche, exploitant plus à fond les procédures d'observation et d'analyse qualitative, se développent autour de nouvelles grilles de lecture du social où sont véhiculées les notions d'autonomie, de réseaux naturels ou de qualité de vie.

Mais ce qui retient le plus notre attention, c'est que l'exploration du quotidien et du vécu des pratiques traduit, non seulement le souci d'une approche plus globale de l'individu et d'une prise en compte des éco-systèmes que constitue son environnement, mais exprime également le besoin d'appréhender le sens et la pertinence des interventions à travers une plus grande complicité des acteurs dans le processus d'observation, d'interprétation et de transformation.

La problématique de l'évolution de la recherche évaluative mériterait à elle seule un examen plus approfondi. Mais notre propos, dans le présent exposé, vise plutôt à :

1. dégager les principaux enjeux qui résultent de ces transformations;

2. présenter les stratégies d'évaluation couramment en usage, notamment les évaluations diagnostique, formative et sommative ;

3. soumettre des pistes à partir desquelles la recherche évaluative serait susceptible d'apporter une contribution significative. 


\section{Problèmes et enjeux de la recherche évaluative}

Récemment, les analyses méta-évaluatives prenant la recherche évaluative comme objet ont dressé des bilans plutôt sombres de la situation. Dans son analyse de quelque cent rapports diffusés pour la plupart au Québec, Gauthier (1982) conclut que près de la moitié des études ne devraient pas être considérées crédibles et que les lacunes les plus évidentes proviennent d'un manque de préparation du terrain et d'une mauvaise structuration de la recherche.

La situation en France paraît identique. Soulignant l'ambiguïté du statut de la recherche évaluative, Sanchez (1984) va même jusqu'à conclure qu'il conviendrait de limiter la portée de l'évaluation à titre de moyen de connaissance utile à l'action. L'auteur fait aussi remarquer que les résultats obtenus et les recommandations qui en découlent, sont assujettis eux-mêmes à des perspectives d'intérêt et d'opportunité aux différents paliers décisionnels, ce qui annule leur validité d'un point de vue strictement méthodologique.

Aux États-Unis, la problématique de l'utilisation de la recherche évaluative est même devenue une préoccupation majeure depuis quelques années (Weinberg, 1979 ; Tarschys, 1983 ; Weiss, 1983). Weiss (1983) signale qu'elle apporte une contribution qui la réduit en définitive à un rôle d'éclaireur critique. Dans cette même veine d'idée, Tarschys (1983), ainsi que Patton (1978) mentionnent que plusieurs observateurs et analystes débouchent à la conclusion que la recherche évaluative, en bout de ligne, n'offre aucune perspective utile pour l'action ou la décision.

Mais pour ouvrir le débat sur ses enjeux et ses problèmes, un cadre de réflexion s'impose. D'entrée de jeu, nous soumettons qu'elle est d'abord et avant tout une pratique politique au double sens du terme, c'est-à-dire non seulement parce qu'elle véhicule des représentations, pose des actions ou effectue des choix qui souscrivent à des idéologies et à différentes lectures du social, mais également parce qu'elle s'inscrit dans un contexte, une dynamique de lutte dont l'enjeu demeure l'appropriation ou le contrôle de l'information et du processus de rationalisation de la gestion. En tant que pratique politique, elle intervient nécessairement dans le rapport de force et de pouvoir liant étroitement les agents situés dans trois sphères principales : le champ de la décision politique et administrative, le champ professionnel de l'action sociale, le champ scientifique de la connaissance. Or, une question se pose. Une recherche évaluative neutre et désintéressée estelle possible dans un contexte où tous les agents concernés, même 
externes, ont, ultimement, avantage à assurer la continuité d'un programme, d'une action? Suchman (1967) et Roskin (1979), étant préoccupés par cette question, croient pouvoir contourner cette contradiction en proposant que l'évaluation combine deux approches, l'une conduite par un chercheur externe dont la tâche consisterait à évaluer si les objectifs d'une action ont été atteints, l'autre, menée "à l'interne", viserait à valoriser les aspects positifs de l'activité réalisée. Mais, à la réflexion, tous les acteurs œuvrant dans ces différents champs ne sont-ils pas susceptibles d'être visés ou confrontés par les résultats de l'évaluation? N'ont-ils pas intérêt à défendre l'espace collectif que constitue l'activité à évaluer? Cette position de problème apparaît préalable à toute tentative visant à comprendre et mieux jauger les réussites et les échecs de la recherche évaluative et, partant, à mieux circonscrire les obstacles qu'elle cherche à surmonter pour affirmer son identité.

En second plan, on conçoit également la recherche évaluative comme pratique scientifique, au sens où son discours se construit à partir de rationalisations conceptuelles et théoriques et qu'elle appréhende la réalité à l'aide de procédés méthodologiques censés se conformer avec les règles établies de l'investigation scientifique. La spécificité du langage et la rigueur représentent des conditions essentielles de légitimation de la démarche de recherche; paradoxalement, elles constituent en même temps des entraves à son pouvoir d'action, du moment qu'elle encourt une rupture des communications, qu'elle opère une distanciation verbale avec les sujets de l'évaluation. Mais que la critique dépasse le seuil du tolérable, il s'en trouvera toujours pour reprocher à l'étude ou au chercheur son manque d'honnêteté, l'absence de rigueur ou une méthodologie fautive.

Dès lors, étant encastrée dans le triple registre de l'administratif, de l'intervention et du scientifique, comment peut-elle espérer contribuer, épistémologiquement parlant, à l'édification d'une théorie de la pratique évaluative? À partir de quels critères, sur la base de quels principes, en référence à quelles instances peut-on déterminer la valeur de cette forme de recherche ? ${ }^{2}$

Il résulte de ce qui précède que la plupart des difficultés affectant l'élaboration, la mise en œuvre et les retombées de la recherche évaluative ont pour origine le rapport de force même structurant les différents champs opérant dans le processus de la décision administrative et de l'intervention sociale. Les facteurs humain et politique jouent certainement un rôle plus important dans l'évaluation que dans tout autre type de recherche. L'information étant source de pouvoir, on conçoit aisément que l'évaluation puisse constituer en soi une source 
de pouvoir politique importante (Guba et Lincoln, 1983). Le contexte d'une activité de cette nature fournit en quelque sorte un lieu, une occasion, où peuvent s'actualiser les confrontations entre agents préoccupés par le contrôle de leur rayon d'action et la défense de leur pouvoir et prérogatives. Ces affrontements se manifestent lors de la définition initiale du mandat de recherche, dans la clarification des objectifs, dans l'interprétation des résultats et surtout lorsque vient le moment de dégager des recommandations.

La difficulté de savoir si les objectifs d'un programme ou d'une action ont été atteints découle donc d'un même processus complexe. Le chercheur doit tenir compte des objectifs manifestes et latents, de l'écart entre les objectifs officiels et ceux réellement poursuivis dans l'action, sans compter les multiples variantes dans l'interprétation des différents niveaux de ceux-ci. Le même scénario des mises au point et aussi des confrontations se répète dès qu'il s'agit d'opter pour une stratégie d'évaluation, d'allouer les ressources nécessaires ou d'arrêter un choix sur un secteur devant être évalué.

Mais ce sont surtout les résistances, ou du moins les attitudes de méfiance qui surgissent en cours de route qui illustrent le mieux le poids des enjeux en présence ${ }^{3}$.

Or, contrairement à une croyance trop répandue, l'évaluation ne représente pas une menace uniquement pour le praticien (Sanchez, 1984) mais aussi pour le gestionnaire (Roskin, 1979) et le chercheur luimême. Il est admis que de nombreux praticiens accueillent avec suspicion le chercheur investi du rôle d'enquêteur des pratiques, ce qui se traduit souvent par une collaboration mitigée lorsque vient le moment de rendre compte du travail accompli, et une faible utilisation des résultats (Rosenblatt, 1968). De son côté, le gestionnaire, n'étant pas exempt de résistances, s'efforcera à l'occasion d'échapper à la critique en bloquant l'accès à certaines données sous prétexte de confidentialité (Renard, 1985). Pour contourner l'obstacle, souvent le chercheur se verra forcé de reconstituer son matériau d'enquête au moyen de données externes recueillies auprès des usagers. Celui-ci se trouve ainsi dans une position insolite du fait qu'il doit, dans maintes situations, jeter un regard critique, porter un jugement sur celui même qui le commandite. Bref, l'évaluateur met toujours en jeu sa compétence à analyser avec rigueur, cohérence et justesse une action ou un projet, étant donné les contraintes inhérentes au milieu, c'est-à-dire les attitudes parfois réfractaires du client ou encore les conditions défavorables (délai imposé, limites budgétaires) dans l'exercice de son mandat. Il subsiste ainsi une certaine incompatibilité dans l'application d'une politique à double standard lorsqu'il s'agit de présenter un 
produit de recherche qui soit directement et immédiatement transposable dans l'action et à la fois scientifiquement sans faille.

\section{Stratégies possibles}

Le débat sur les problèmes et les enjeux de la recherche évaluative n'est certes pas clos. Toutefois, dans la perspective de le poursuivre, il apparaît important de dégager les fondements et les objectifs des principales pratiques d'évaluation, en lien avec ce qui précède. Sans prétendre brosser un tableau exhaustif de la situation, nous proposons une nomenclature regroupant sous trois principaux types les stratégies d'évaluation : diagnostique, formative et sommative.

\section{L'évaluation diagnostique}

On qualifie de diagnostique une évaluation lorsque, située en début d'un processus d'intervention ou d'implantation de programme, elle dresse l'inventaire des besoins de la clientèle et des ressources disponibles du milieu. Elle constitue donc une étape descriptive, une opération de dépistage conduisant à une planification appropriée de services, de programmes ou de stratégies d'intervention.

Ce diagnostic de situation (à ne pas confondre avec la conception médicale de "diagnostic»), renvoie à une démarche essentielle d'exploration, qui peut être à la fois empirique et théorique. L'exploration empirique s'attache principalement aux conditions de réalisation d'une action sur le terrain, soit les attentes d'une clientèle, d'un groupe ; bref, ce qui fonde habituellement l'action. Vue sous cet angle, elle correspond à une étude de besoins combinant une démarche d'inventaire (cueillette de données) et une de confrontation des données recueillies avec les groupes ou clientèles concernées. Dans ce type d'exploration, le chercheur joue souvent un double rôle: celui d'éclaireur critique ou "d'expert", et celui d'observateur participant soucieux de saisir le pouls de la population étudiée.

L'exploration théorique s'attache plutôt aux conditions d'action en terme de faisabilité. Essentiellement, l'optique est d'estimer dans quelle mesure l'évaluation paraît possible et viable dans un contexte donné. Parmi les questions soulevées, on retrouve celles-ci: Quels sont les moyens et les ressources en place? Quel écart existe-t-il entre les fins recherchées et les moyens? Quelles sont les meilleures stratégies d'action possibles eu égard aux limites identifiées? 
Une fois attribué le sens à la notion d'évaluation diagnostique, il convient d'expliciter plus à fond chacune de ses composantes.

\section{L'étude de besoins}

La notion de besoin fait partie du discours quotidien, particulièrement celui des travailleurs sociaux. Toutefois, on ne peut parler d'univocité dans les définitions courantes de ce concept. D'après Plante (1983) : «le Dr Bise (1968) relève 27 sortes de besoins dans la liste de Garnier (1852), 12 dans celle de Ribot (1917), 26 chez Drever (1977), 14 chez Dumas (1937), 44 chez Mc Dougall (1935), 38 chez Dunlap (1934-35), 31 chez Murray, etc... " Cette surutilisation du concept explique et maintient la confusion des objectifs poursuivis par ceux qui réalisent des inventaires et des analyses de besoins.

Tantôt on l'associe au corporel, au physiologique, tantôt au psychologique, au spirituel ou au monétaire. Que l'on se réfère à la pyramide des besoins élaborée par Maslow (1972), à la typologie proposée par Saint-Arnaud (1974) ou à d'autres, il subsiste un consensus autour des caractéristiques intrinsèques à cette notion, soit: la nécessité, la plasticité, la subjectivité et l'interdépendance entre les différents niveaux de besoin.

Tyler (1950) a proposé une définition opérationnelle utile aux évaluateurs : le besoin représente l'écart entre une situation actuelle problématique et une situation désirée. Il signifie donc une différence perçue, exprimée et mesurée entre ce qui est (situation actuelle) et ce qui devrait être (situation idéale) (Kaufman, 1972).

Cette définition comporte ceci de particulier : elle incorpore une dimension objective et une dimension subjective. Il existe donc une recherche au niveau de l'expression du besoin et des meilleurs moyens pour y parvenir, et une autre au niveau de la mesure qui peut s'avérer nécessaire selon les situations.

L'inventaire des besoins peut se mener, méthodologiquement, de plusieurs façons: selon un modèle inductif, déductif ou mixte. L'élément de choix entre un modèle ou un autre dépend des habiletés, des possibilités et des objectifs de celui qui entreprend cette évaluation.

Adopter une approche inductive, c'est postuler que les groupes, organismes ou individus qui vivent des difficultés sont les meilleurs informateurs, d'où la nécessité de rassembler des informations à l'état naturel sur le vécu d'une situation donnée. En revanche, l'approche déductive, même si elle requiert de rejoindre les populations cibles, recueille ses informations suivant un canevas préétabli. La population 
ou les sous-groupes concernés sont alors interrogés dans une perspective de validation d'hypothèses ou d'éléments de contenus prédéterminés. Quant à l'approche mixte, elle intègre certains aspects des deux démarches précédentes.

Historiquement, c'est l'approche déductive qui a prévalu. En même temps que s'animait en recherche le débat entre les méthodes quantitatives et les méthodes qualitatives, l'approche déductive, qualifiée de plus scientifique et rigoureuse parce que faisant davantage appel aux mathématiques, s'affirmait de plus en plus. On doit à cette approche d'avoir mis en évidence une multitude de sondages et d'études sur les besoins de la population, qui ont inspiré par la suite de nombreux chercheurs. Tel fut le cas, à titre d'exemples, de l'étude menée par Beaudoin et al. (1972) dans la région de l'Estrie sur les besoins des personnes âgées, et celle de Martin (1963) sur le logement.

Depuis quelques années, cependant, l'approche inductive connaît un nouvel élan, surtout avec la vogue du courant de la recherche qualitative. Conséquemment, la perspective d'évaluation diagnostique tend à se modifier: elle devient moins formaliste, plus axée sur l'introspection, plus holistique, plus naturaliste; bref, au sens où l'entend Lecomte (1982), plus normative qu'empirique, c'est-à-dire centrée davantage sur les valeurs que sur des ensembles de lois ou de faits.

Dans cette recrudescence du courant dit naturaliste se dessine une approche plus subjective et généralement mieux acceptée des praticiens sociaux qui sont impliqués dans de telles recherches.

Cependant, peu importe le type d'approche privilégié en fonction des limites spécifiques à chaque milieu, ce qui apparaît fondamental dans une évaluation de type diagnostique ce sont les principes suivants :

- on n'élabore pas un programme ou un plan d'intervention sans une connaissance approfondie des éléments qui composent une situation problématique (diagnostic de situation);

- l'évaluation diagnostique doit préférablement être réalisée par un évaluateur externe à la gestion éventuelle d'un programme ; le focus doit être mis sur les besoins réels tels qu'exprimés plutôt que sur une programmation de services existante ;

- l'analyse des besoins doit être saisie comme étant indépendante du processus décisionnel ;

- les besoins inventoriés ne sont jamais que des expressions de besoins formulés par des agents divers, pour eux-mêmes ou pour d'autres; en cela, la notion de besoin est subjective et plastique ; 
- toute méthodologie est justifiable en autant qu'elle tienne compte du type d'informateurs à consulter, de leurs effectifs, du genre d'informations recherchées, de la facilité avec laquelle ces informations pourront être traitées et des ressources actuellement disponibles.

Une telle évaluation diagnostique est donc particulièrement utile à l'étape de la planification d'un programme ou de l'élaboration d'une stratégie d'intervention. Elle correspond à l'étape de la position du problème dans un modèle empirique de recherche. Toutefois, il demeure également possible de poser un diagnostic de situation en cours d'intervention ou de déroulement d'un programme; on rejoint alors la perspective de l'évaluation formative qui sera traitée plus loin.

\section{L'étude de faisabilité}

La deuxième facette de l'évaluation diagnostique porte sur la faisabilité, c'est-à-dire l'examen des conditions entourant les possibilités de mise en ouvre de l'action, qu'il s'agisse d'une politique, d'un programme ou d'une intervention professionnelle. Jusqu'ici, eu égard à la planification de l'action, la tendance a été forte d'ignorer l'importance de la recherche, en particulier celle de l'évaluation. En fait, que ce soit pour la formulation d'une politique, pour l'élaboration d'un programme ou plus modestement pour l'établissement d'un plan d'intervention, il est insuffisant d'identifier les besoins et de construire un plan d'action; encore convient-il d'être en mesure de savoir si l'action pourra effectivement être réalisée. Trop souvent a-t-on eu tendance à définir les objectifs et à prendre les décisions sans une bonne connaissance préalable des facteurs susceptibles d'influencer la réalisation du projet (Mayer et Greenwood, 1980: 55-60).

En définitive, un plan devient réalisable quand il est pratique et correspond aux conditions de la réalité, en d'autres termes quand «il colle à cette réalité ". Il implique en même temps l'obtention du support de ceux qui sont concernés ou touchés par le programme. L'utilisation de l'évaluation dès le moment de l'élaboration du plan d'action s'avère un moyen de s'assurer que ces conditions sont satisfaites.

L'évaluation de la faisabilité comprend trois volets principaux :

1) elle vise en premier lieu à établir quels sont, parmi les objectifs possibles, ceux qui apparaissent le plus facilement et le plus aisément réalisables ;

2) elle s'adresse ensuite à l'estimation du potentiel des ressources existantes pour l'atteinte des objectifs prévus; 
3) elle sert finalement à établir jusqu'à quel point l'action ou les actions envisagées vont recevoir le support du milieu ou de ceux qui sont immédiatement concernés.

En ce qui regarde le premier volet, l'étude se centre sur la description aussi précise que possible des objectifs, en tenant compte de la réalité dans laquelle s'insèrent la politique, le programme ou l'intervention (Wholey, 1981). On pourra fournir un estimé du degré de succès qu'on peut attendre de telle ou telle alternative d'action une fois celle-ci en opération. Il faut déplorer que trop souvent des programmes ou des interventions soient mis en opération alors qu'ils sont trop faibles pour produire les effets escomptés (Sechrest, West, Philips, Redner et Yeaton, 1979).

Elle fournit également, dès l'étape de la planification, les moyens de connaître les possibilités qu'une politique, un programme ou une intervention soient mis en place et fonctionnent adéquatement selon un ensemble de critères déterminés eu égard aux ressources nécessaires. Par le fait même, elle permet de mieux connaître quels types de ressources humaines ou autres sont nécessaires à l'exécution du plan, si les ressources en place sont suffisantes, si le personnel est adéquatement préparé, si ce qu'on est en train d'élaborer utilise au mieux les ressources dont on dispose.

L'établissement de la faisabilité vise aussi, en troisième lieu, à déterminer si le milieu de même que les cibles visées par le programme sont prêts à accepter, à supporter ou même à s'impliquer ou à participer aux orientations proposées. Quelles sont les attitudes du milieu par rapport aux actions envisagées? Sera-t-il prêt à coopérer dans la réalisation du programme? Peut-on anticiper des résistances imprévues? $\mathrm{Y}$ aura-t-il des déviations relativement aux objectifs initiaux, compte tenu des diverses conceptions des personnes impliquées? Voilà autant de questions auxquelles s'adresse l'évaluation de la faisabilité.

Les méthodes disponibles pour l'évaluation de la faisabilité n'ont pas été, jusqu'à présent, très élaborées. Elles sont avant tout exploratoires et descriptives. Il faut néanmoins souligner l'utilité et les possibilités de la méthode développée par Edwards, Guttentag et Snapper (1975) puis reprise de manière plus complète par Edwards (1977). Celle-ci, dite d'utilité à attributs multiples, (MUAM ou, en anglais, the multi-attribute utility method-MUAT) est une technique inspirée de la théorie de la décision qui, tout en constituant un guide pour la prise de décision, présente l'avantage de se fonder sur l'évaluation pour établir les domaines où elles doivent être prises. Les principales caractéristiques de la méthode (Posavac et Carey, 1985 : 108-118) sont de décomposer le processus, c'est-à-dire de séparer les 
éléments d'une décision complexe et de fournir une évaluation de chaque élément isolément. Au lieu de se concentrer sur la décision d'une manière globale et d'amorcer une discussion d'ensemble à propos des mérites relatifs des différentes lignes d'action les unes par rapport aux autres, l'évaluation se centre sur l'aide à apporter aux responsables sur ce qu'ils essaient d'accomplir au moyen de diverses lignes d'action, ou, en d'autres termes, au moyen de divers éléments du programme. Ainsi, on essaie, grâce à cette méthode, de déboucher sur une entente sur les objectifs d'ensemble à réaliser, pour ensuite établir un consensus sur les critères à utiliser pour l'établissement des lignes d'action les unes par rapport aux autres. Pour la planification, cette approche constitue une façon de circonscrire les sources de désaccord existant entre les planificateurs ou les preneurs de décisions, et de mettre l'accent sur les zones où l'accord peut être obtenu.

En somme, l'étude des besoins et celle de la faisabilité se conjuguent pour que la planification de l'action corresponde le plus fidèlement possible aux attentes d'une population donnée, selon les ressources disponibles. C'est ainsi que l'action à réaliser est définie et formulée dans une optique réaliste, tenant compte des ressources nécessaires et disponibles, et enfin pour qu'elle puisse être mise en place en tenant compte de la nécessaire collaboration, coopération et implication du milieu à sa réalisation.

\section{L'évaluation formative}

On peut concevoir l'évaluation formative à deux niveaux, soit dans le cadre de l'évaluation ou d'un programme ou d'une intervention. Bien que les fondements puissent être communs, il existe une différence quant à l'objectif poursuivi.

\section{L'évaluation formative de programme}

Dans le contexte de l'évaluation d'un programme, il est possible et même recommandé d'étudier, non seulement les résultats ou l'efficacité d'un programme d'intervention ou de services, mais le processus qui conduit à des résultats, escomptés ou non. L'évaluation formative a cette particularité de s'inscrire pendant le déroulement d'un programme; elle vise principalement à questionner le processus, c'est-à-dire les moyens mis en place pour l'atteinte des objectifs. Elle y joue donc principalement à l'intérieur du processus un rôle de régulation, qui entraîne des ajustements jugés nécessaires dans la 
poursuite de l'implantation ou de la réalisation d'un programme. Sans ce correctif, on ne peut parler d'évaluation formative.

Si l'on se réfère à quelques auteurs bien connus en matière d'évaluation, on se rend compte que ni Tyler (1967), ni Hammond (1980), ni Metfessel et Michael (1967), ni Provus (1971) ne se sont préoccupés de cette dimension. En effet, Tyler, qui visait uniquement la mesure du degré d'atteinte des objectifs, se limitait à comparer des performances entre un temps "avant" et un temps "après" le programme. Aucune mesure "pendant " n'étant prévue, il ne se souciait donc aucunement des correctifs à y apporter et, par conséquent, il ne s'agissait pas en soi d'évaluation formative. Dans ce contexte, il est évident que l'on discutait d'évaluation de programme, sans qu'il soit véritablement question du programme en soi. Rossi (1972) illustre très bien ce courant historique lorsqu'il évoque la "boîte noire" de l'évaluation. On questionne les inputs, on mesure les outputs, mais on ne s'attarde pas sur le processus comme tel.

Hammond (1980) a toutefois avancé l'idée d'analyse de résultats consécutifs à une mesure par interaction de facteurs, ce qui est très intéressant en soi puisque l'on peut combiner diverses dimensions et raffiner l'interprétation. Cependant, il appliquait ses instruments une fois le programme terminé, de sorte qu'on peut qualifier sa démarche d'exclusivement sommative.

Metfessel et Michael (1967), de leur côté, ont ajouté un élément intéressant dans leur modèle, à savoir celui de l'administration périodique des instruments de mesure. On croirait alors qu'ils initiaient la dimension formative en évaluation de programme, mais tel ne semble pas être le cas. En effet, leurs mesures à séries chronologiques n'étaient qu'une addition de mesures sommatives compilées en une seule à la fin du programme. Ils rejoignaient d'ailleurs Provus (1971) sur ce point, qui définissait son "processus» comme l'évaluation de l'atteinte des objectifs intermédiaires.

Les auteurs qui s'inscrivent donc carrément en évaluation formative sont: Stake (1967), Alkin (1969) et Stufflebeam (1980). Stake met l'accent sur la congruence entre les intentions (programme théorique) et les observations (programme vécu). Il évalue ou juge cette congruence à partir de deux types de standards (absolus et relatifs). Ce que nous en retenons, c'est donc, d'une part, l'idée d'évaluation formative et, d'autre part, l'utilisation de standards absolus pour porter un jugement.

Pour Alkin, il y a évaluation formative quand on évalue la congruence entre la planification et l'implantation et, surtout, quand on se permet d'intervenir soit au niveau du plan, soit au niveau de 
l'implantation dans le but d'apporter un correctif à un problème identifié en cours de processus.

Stufflebeam, de son côté, y accorde une tout aussi grande place. Dans son "processus", il réfère à la mise en place, au contrôle et au raffinement du plan; dans son "produit » il renvoie à la relance, c'est-àdire à la mise en relation des résultats du programme avec les objectifs, les intrants et le processus. Stufflebeam est même beaucoup porté sur le formatif et peu sur le sommatif. Son processus de changement planifié implique qu'un programme n'est jamais terminé et que subsiste toujours la possibilité d'une interprétation-relance.

L'évaluation formative de programme doit donc être conçue principalement comme un mécanisme de régulation. Comme l'exprime Rutman: "par opposition aux évaluations de l'efficacité qui portent un jugement sur les programmes, la recherche formative se met à leur service. [...] La recherche formative met donc l'accent sur la découverte de résultats, tandis que les évaluations de l'efficacité s'intéressent principalement à la vérification. » (Rutman, 1982: 67).

Bref, ce type d'évaluation est approprié pour vérifier la congruence entre les caractéristiques et les besoins spécifiques d'une clientèle et les objectifs, contenus et moyens véhiculés dans le cadre d'un programme d'intervention. Le programme touche-t-il les bonnes cibles? Les interventions sont-elles adaptées ? etc.

Sur le plan méthodologique, les évaluations formative et sommative se distinguent par le fait que la première recherche des informations, tant subjectives qu'objectives, nécessaires à la compréhension du vécu du programme, plutôt que des résultats. En ce sens-là, elle requiert une approche inductive, c'est-à-dire basée sur la compréhension des phénomènes à évaluer dans leur état naturel, ces informations à recueillir pouvant revêtir différentes formes : observations concrètes sur le terrain, témoignages, analyse de contenu, de documents, etc. Elle peut donc s'inspirer à la fois des méthodes qualitatives et quantitatives ${ }^{4}$.

Cardinet (1985), dans un article récent, stipulait que deux approches d'évaluation formative peuvent être empruntées: I'une est appelée analytique et l'autre, globalisée. L'évaluation analytique se conçoit en observant les progrès dans la perspective de l'analyse des réussites, des déficits et des erreurs. Quant à l'évaluation globalisée, elle consiste à repérer l'origine des difficultés vécues dans le programme par l'étude du fonctionnement d'ensemble. L'intention ici n'est pas de comprendre chaque situation difficile en détail, mais de déterminer une stratégie de remplacement qui ne bute pas sur les mêmes difficultés mais qui, au contraire, s'appuie sur les points forts du programme. Bref, 
il s'agit d'une évaluation qui permet à une unité et aux intervenants de se réajuster et d'améliorer les services dispensés. Dans ce sens-là, il s'avérerait souhaitable que ce soient les intervenants concernés qui soient les initiateurs. L'évaluation formative se conçoit difficilement sans une appropriation du sens et des outils de l'évaluation par ceux qui sont responsables du déroulement d'un programme.

\section{L'évaluation formative de l'intervention}

Bien que les postulats de base de l'évaluation formative soient identiques dans le contexte de l'intervention, il est clair qu'ici on interpelle l'intervenant dans sa pratique. Dans un contexte social où l'évaluation est perçue comme mécanisme de gestion et de contrôle, les intervenants sont peu portés à en utiliser les outils pour jeter un regard critique sur leur pratique.

À l'instar de l'évaluation de programme, l'évaluation de l'intervention vise la régulation par la rétroaction qualitative. Allal $(1979,1981)$ et Cardinet (1985) ont par ailleurs exploré deux formes de régulation : la régulation rétroactive et la régulation proactive.

Dans le cas d'une régulation rétroactive, on cherche des moyens pour remédier aux problèmes qui ont surgi dans une intervention, compte tenu de l'objectif visé. La notion de régulation proactive, quant à elle, implique qu'on cherche à adapter et à différencier des modes d'intervention future de façon à consolider les compétences. Nous abordons ici la notion de " profil " d'intervenant ou profil d'intervention. Chaque intervenant possède son style propre, ses points forts et ses faiblesses, de sorte que l'observation de ces caractéristiques, dans le cadre d'une évaluation formative, permet d'en dégager le profil, ses impacts et les mécanismes d'ajustement appropriés.

Sur le plan méthodologique, plusieurs outils sont utilisables par les praticiens, allant de l'étude de cas à d'autres techniques comme celle de l'incident critique, l'analyse d'interventions enregistrées, etc ${ }^{5}$.

Sans qu'il soit utile de pousser plus loin cette analyse, il y a lieu cependant d'émettre certaines hypothèses quant à l'utilisation de la recherche évaluative, particulièrement dans un but formatif. Les praticiens sociaux l'associent vraisemblablement aux mécanismes de gestion et de contrôle des services et en font, de façon générale, peu de cas (Rosenblatt, 1968 ; Shaw et Walton, 1978; Raynor, 1986). Bien qu'ils ne s'engagent eux-mêmes que rarement dans ce type d'activités, on ne peut pas conclure pour autant qu'ils n'évaluent pas leur intervention. Toutefois, lorsque des remises en question des modes d'intervention sont entreprises, elles le sont par l'intermédiaire d'une recherche dite 
"recherche-action " 6 . Faut-il en conclure qu'il s'agit là d'une approche plus acceptable, ou tout simplement plus naturelle pour les praticiens de questionner leur pratique? Dans l'affirmative, ne devons-nous pas, en tant que chercheurs, nous empresser d'examiner nos propres méthodes de recherche évaluative?

\section{L'évaluation sommative ou des résultats}

Historiquement, l'évaluation "sommative" ou des résultats est d'abord celle qui s'est développée. D'ailleurs, encore aujourd'hui, c'est cette forme qui demeure au cour des préoccupations en la matière et, bien souvent, lorsqu'on utilise le concept d'évaluation, c'est à elle qu'on réfère en premier lieu.

L'évaluation sommative cherche à établir si une action produit les résultats ou les effets escomptés. Comme l'a fait ressortir Freeman (1977), on peut remonter aussi loin que dans les années trente pour retrouver des chercheurs en sciences sociales qui ont insisté sur l'utilisation des méthodes de recherche afin d'établir si les programmes produisent les résultats anticipés et atteignent les objectifs visés. Vers la fin des années cinquante sont apparues les premières entreprises systématiques d'évaluation dans le domaine des programmes sociaux. C'est à cette époque qu'on a commencé à utiliser, sur une base régulière, des techniques de recherche pour connaître les résultats des actions entreprises en santé publique, dans le domaine de la délinquance et de la criminalité, ou pour établir le niveau de succès atteint par les différentes thérapies. Au cours des années soixante, l'utilisation des méthodes de recherche en évaluation devint une réalité beaucoup plus fréquente, comme l'atteste l'ouvrage de Suchman (1967). Mais c'est à partir du début des années soixante-dix qu'on assiste véritablement à la formalisation de la méthodologie concernant l'évaluation des résultats avec la prolifération des publications sur le sujet (Caro, 1971; Rossi et Williams, 1972; Weiss, 1972). De plus, l'introduction dans l'administration publique d'une budgétisation par programme et de politiques en matière d'évaluation, surtout au niveau fédéral, ont contribué à rendre l'évaluation sommative de plus en plus présente dans les programmes entrepris, même si les résultats n'ont pas toujours été à la hauteur des attentes.

On peut examiner l'évaluation sommative suivant ses orientations traditionnelles centrées sur une définition formelle des programmes, et à la lumière des nouvelles tendances qui se sont fait jour au cours des dernières années: l'approche qualitative et son rapprochement de l'intervention. Telle est la double perspective adoptée ici. 
Les orientations traditionnelles:

une démarche d'évaluation centrée sur une définition formelle du programme

L'évaluation des résultats s'est couramment faite, jusqu'à maintenant, selon un processus qui suppose l'existence et la définition d'une action sous la forme d'un programme bien articulé et ayant des objectifs bien déterminés. Essentiellement, la démarche présuppose d'abord que celui-ci satisfait à des critères d'évaluabilité, c'est-à-dire que ses objectifs sont définis clairement et de manière mesurable, que les liens existant entre les activités réalisées et les résultats attendus sont explicités selon une logique qui se fonde sur l'état des connaissances et que la structure du programme est définie de manière claire et articulée.

Prenant pour acquis l'existence d'un programme formellement élaboré, l'évaluation est une entreprise externe réalisée selon les critères de la méthode scientifique. Elle vise à vérifier si les résultats produits correspondent aux objectifs fixés, en utilisant un ensemble de critères définis de l'extérieur, les critères de succès du programme. Plus précisément, il s'agit d'en arriver, par des méthodes de recherche fiables et valides, à établir si les effets constatés sont causés par le programme en éliminant ou en contrôlant tous les autres facteurs externes ou internes qui pourraient influencer les résultats.

Ces facteurs sont nombreux et plusieurs auteurs s'y sont intéressés (Campbell et Stanley, 1966 ; Cook et Reichardt, 1979 ; Lecomte et Rutman, 1982 ; Rossi et Freeman, 1985). On retrouve parmi eux les changements liés à l'histoire ou à la maturation, les éléments d'interférence qui se substituent au programme ou les variables imprévues; d'autres sont plutôt attribuables au type de devis ou aux instruments de mesure utilisés, à une contamination propre ou liée aux conditions de réalisation du programme, etc. Ce ne sont là que quelques-uns des facteurs qu'on essaie de contrôler en utilisant un processus de recherche qui soit aussi indépendant que possible de l'action en fonction des critères externes qui auront toutes les chances de correspondre aux canons classiques de la méthode scientifique selon l'approche hypothético-déductive.

Les protocoles de recherche utilisés dans ce type d'évaluation doivent être définis de manière à tenir compte, le plus possible, des critères rigoureux de la méthode scientifique, en utilisant, somme toute, comme modèle de référence idéal, les protocoles expérimentaux avec échantillons choisis de façon aléatoire. Comme, bien entendu, une telle procédure n'est pas toujours possible, on pourra recourir à 
des protocoles de nature quasi expérimentale, à l'analyse de séries chronologiques, à l'analyse de régression sur des séries chronologiques multiples, etc. Toutes ces méthodes, et en particulier celles qui font appel à des protocoles non expérimentaux (études avant-après, études rétrospectives, etc.) sont définies comme des compromis plus ou moins acceptables par rapport à la « vraie » méthode de l'expérimentation avec échantillonnage aléatoire.

Malgré leurs avantages, de tels protocoles ont été l'objet de plusieurs critiques au cours des dernières années. De plus en plus, on remarque une insatisfaction grandissante envers l'utilisation de protocoles de recherche aussi formels en matière d'évaluation. En même temps, leurs résultats n'ont pas toujours été très concluants, surtout en matière de programmes sociaux. On les attaque parce que trop séparés et isolés de l'intervention et des intervenants. Ces derniers éprouvent de la difficulté à en saisir la pertinence, d'autant plus que leur réalisation s'est souvent faite de manière isolée sans implication réelle et effective des premiers concernés dans le processus de recherche. De plus, plusieurs entreprises d'évaluation ont été plus ou moins irréalistes quant à leur ampleur; elles ont produit des résultats dont la portée était somme toute assez limitée et leurs résultats ont été peu utilisés dans la pratique.

Tendances récentes: l'insertion des méthodes qualitatives et liaison plus étroite avec l'intervention

Au cours des dernières années, de nouvelles orientations en la matière se sont développées. Sans remettre complètement en cause les accomplissements réalisés précédemment, elles n'en constituent pas moins des réponses, au moins partielles, à certaines des limites constatées et aux critiques adressées à l'évaluation telle que présentée précédemment. Ces orientations sont regroupées autour de deux pôles principaux: celui de l'approche qualitative et celui des liaisons entre l'évaluation et l'intervention.

a) L'insertion de l'approche qualitative dans l'évaluation des résultats

Comme on l'a vu précédemment, en parlant de l'évaluation diagnostique où elle s'est d'abord développée, l'évaluation qualitative est moins formaliste, plus axée sur l'introspection, plus holistique et 
plus naturaliste. Pour évaluer les résultats, elle ne part plus d'une définition formelle des objectifs du programme, mais considère plutôt ce dernier comme un tout, et c'est à partir de ce tout que le processus s'enclenche (Patton, 1980). Son approche est inductive en ce sens que l'évaluation essaie de faire l'analyse des résultats du programme à partir de la situation du programme vue en elle-même et non pas à partir de la seule conception des objectifs planifiés dont on essaie de vérifier la réalisation dans le fonctionnement quotidien. Elle est en même temps naturaliste, en ce sens qu'elle prend l'action en tant que telle et qu'elle tente de s'en rapprocher et de la comprendre de l'intérieur, plutôt que de manipuler le phénomène de l'extérieur.

Sur le plan méthodologique, l'approche qualitative procède à partir de deux techniques principales, bien connues en anthropologie : l'observation et l'entrevue. Ce sont les deux grandes méthodes par lesquelles il est possible à l'évaluateur de faire la collecte de données qualitatives en vue de faire l'analyse des résultats du programme à partir de son fonctionnement direct, sans lui greffer un schéma d'analyse de type hypothético-déductif.

En fait, trois stratégies de recherche ont été identifiées par Patton (1980) comme plus particulièrement pertinentes à l'évaluation qualitative. La première est celle du modèle transactionnel, qui insiste sur le maintien d'une interrelation continuelle entre les participants ou responsables du programme et les évaluateurs, pour que l'information soit utilisée au fur et à mesure qu'elle est recueillie dans une sorte de mouvement d'aller-retour entre chercheurs et intervenants. La deuxième se centre autour du modèle qu'on appelle "libre objectif ", c'est-à-dire qui ne part d'aucune définition des objectifs du programme, mais qui l'observe dans son fonctionnement ou interroge les participants pour en découvrir les résultats à partir des données ainsi recueillies. En dernier lieu, on retrouve le modèle centré sur la prise de décision à partir de la connaissance des résultats: il s'agit, au fur et à mesure que doit être prise une décision concernant une action ou un programme, d'obtenir ou de recueillir toutes les informations et les données sur les résultats accomplis qui sont nécessaires à la nouvelle décision à prendre.

Dans l'état actuel des choses, l'approche qualitative, d'origine plus récente, n'est pas encore très développée si on la compare à l'évaluation sommative, conduite suivant l'approche hypothéticodéductive. Elle prend néanmoins de plus en plus d'importance, d'autant plus qu'elle introduit plusieurs changements dans la conception traditionnelle de l'évaluation. Elle suppose une relation bien différente entre l'évaluateur, le responsable de programme et les 
intervenants parce qu'elle s'insère beaucoup plus étroitement et directement dans le programme et se fait à l'aide d'un protocole de recherche qui se construit au fur et à mesure, selon une démarche inductive plutôt qu'un protocole de recherche prédéfini selon l'approche hypothético-déductive. Elle fournit aussi, à notre point de vue, une réponse aux objections et aux difficultés du premier type d'évaluation des résultats et, en particulier, à son impuissance à utiliser des méthodes appropriées d'évaluation de l'intervention en service social (Crane, 1976). Son apport paraît d'autant plus important qu'elle peut être souvent combinée avec l'approche traditionnelle dans la pratique pour en utiliser les avantages respectifs.

\section{b) L'insertion de l'évaluation dans l'intervention}

Devant l'ensemble des critiques adressées aux professions d'aide, et en particulier à l'intervention en service social, quant à leur inefficacité ou à leur absence de préoccupation quant à la mesure des effets de l'intervention, on en est venu à s'interroger sur les moyens de rapprocher l'évaluation et d'impliquer plus activement les praticiens dans cette démarche systématique. Le courant de l'évaluation qualitative dont nous venons de parler et de la recherche-action, dont les différentes significations ont été explorées par Gauthier (1984), a aussi contribué à cette nouvelle orientation.

Depuis longtemps déjà, en service social, on connaît et on a utilisé les études, analyses et révisions de cas ; mais ces méthodes manquent de systématisation et, malgré leurs contributions évidentes qu'il faut surtout éviter de sous-estimer, elles ne sont pas faites dans une perspective d'évaluer les résultats de l'intervention. Au cours des dernières années, il y a eu prise de conscience de la nécessité d'insérer des techniques d'évaluation en étroite relation avec le processus d'intervention, et même de les insérer dans ce processus. Cette insertion a d'abord pris la forme de l'introduction de techniques spécifiques qui s'adressent directement aux résultats de l'intervention, comme celle de l'Échelle d'atteinte d'objectifs (E.A.O.) (Goal Attainment Scaling-G.A.S.) qui s'est développée pour mesurer le degré d'atteinte des objectifs de l'intervention en santé mentale (Kiresuk et Garwick, 1979), mais qui est aussi utilisable dans d'autres types d'évaluation. Pour les approches de modification de comportement, qui sont fondées sur les théories behavioristes, les techniques d'évaluation appliquées à la réalisation des objectifs de changement à réaliser, font partie intégrante de l'intervention. De la même manière, dans le domaine des sciences de la gestion, la gestion par objectifs; qui 
s'est aussi développée pendant la même période, se fonde elle aussi sur une liaison entre la détermination d'objectifs dans la gestion et l'évaluation de leur atteinte par le gestionnaire.

C'est ainsi que l'évaluation est considérée de plus en plus en liaison étroite avec l'action. Depuis la fin des années soixante-dix et le début des années quatre-vingt, aux États-Unis et au Canada anglais d'abord, et plus récemment au Québec, elle a été jointe au processus même de l'intervention grâce à la méthode d'évaluation sur système unique que Beaudoin décrit plus en détail dans un autre article de ce numéro. Dans cette perspective, l'évaluation devient partie de la pratique et elle est intégrée à l'intervention elle-même, comme l'ont fait ressortir clairement un certain nombre d'auteurs (Muchsler, 1979; Bloom et Fischer, 1982; Zimbalist, 1983; O'Brien, 1985). Ainsi, l'évaluation des résultats peut être réalisée par le praticien lui-même, celui-ci devenant évaluateur dans le cadre même de son intervention. À l'aide d'instruments disponibles, comme ceux développés par Hudson (1982) ou tout simplement à l'aide d'instruments construits par les évaluateurspraticiens, les résultats sont évalués en cours d'intervention, en rendant ainsi les constatations immédiatement utiles pour l'action 7 . II va sans dire que cette façon de procéder n'est pas exempte des limites inhérentes à une recherche portant sur un cas unique; mais il s'agit possiblement de l'approche qui paraît la mieux adaptée dans l'état actuel des connaissances en matière d'évaluation.

\section{Jalons d'une conception renouvelée de la recherche évaluative}

Au terme de cet exposé, on conçoit aisément qu'il n'existe pas de modèle-type établi en la matière. Toutefois, du questionnement de fond qui se dégage dans l'ensemble des contributions de ce numéro, et à la lumière des expérimentations nouvelles qu'on a pu observer sur le terrain, notre réflexion nous a conduit à identifier un certain nombre de principes directeurs pouvant servir d'amorce dans l'implantation de futures stratégies en évaluation.

Selon nous, une action renouvelée de la recherche évaluative passe par le développement d'un consensus véritable autour des objectifs qu'elle poursuit, l'aménagement en profondeur des conditions plus propices à l'exercice de son rôle et l'adoption de schèmes de valeurs et d'attitudes assurant la transparence et l'authenticité de la démarche. À défaut de tels principes, subsiste le 
risque d'une mise en doute de l'objectivité de la recherche sociale, de l'accentuation des clivages au sein des groupes d'intervenants, d'oppositions entre connaissance, action et décision et, possiblement, d'une dévalorisation de la recherche sociale elle-même.

\section{- Premier principe : reconnaître la fonction éducative et d’apprentissage de la recherche évaluative.}

Une première condition est de fournir aux participants un encadrement leur permettant de démystifier le rôle et les finalités de la recherche évaluative, en identifiant le système d'action, les enjeux et les problèmes dans lesquels elle s'inscrit. Cette dimension pédagogique, s'effectuant dans un climat de libre-échange et de non-directivité, vise à sensibiliser aux limites de la recherche évaluative et à l'apprentissage des différentes stratégies de recherche. En cours d'exécution d'une activité d'évaluation, la fonction éducative intervient à chacune des étapes du processus, de manière à favoriser la réflexion et inciter à tirer les leçons de l'expérience acquise.

- Deuxième principe : s'assurer que la recherche évaluative devienne une entreprise participative et démocratique.

Tous les intéressés ou participants devraient s'approprier le contrôle de l'ensemble du processus de recherche (définition du mandat et des objectifs, aspects logistiques, observation, analyse et interprétation). II s'agit d'une condition essentielle pour atténuer les clivages, réduire les tensions et la méfiance résultant d'une évaluation externe. La formule associative constitue en quelque sorte un écran protecteur contre toute critique mal éclairée, puisque l'ensemble des participants s'investit du pouvoir d'agir et de décider en connaissance de cause, dans un cadre où l'information puisse circuler librement.

- Troisième principe : concevoir la recherche évaluative comme outil d'analyse, de dialogue et de confrontation.

L'analyse collective des données d'évaluation, débouchant sur des bilans périodiques, doit permettre de rapprocher de façon constructive les différents intervenants et favoriser leur engagement. Le programme ou l'action évaluée devient en quelque sorte un système ouvert, transparent, un terrain privilégié pour susciter un débat sur les finalités et les modalités de l'action entreprise. Cette condition serait-elle exclue, l'évaluation risquerait du coup d'être une simple opération routinière, découpée en séquences programmées, sans qu'on puisse la soumettre facilement à l'examen critique. 
- Quatrième principe: concevoir la recherche évaluative comme outil de changement.

Une recherche évaluative peut éventuellement conclure à l'absence d'efficacité ou d'efficience d'une action. Pour cette raison, le milieu d'action et de la décision doit être préparé à accepter des modifications ou des corrections de parcours, sans que le fardeau de l'échec repose sur un groupe ou une cible en particulier. L'adoption d'une attitude ouverte au changement suppose en définitive que tous s'approprient le sens de l'évaluation, que les lacunes identifiées engagent la responsabilité de l'ensemble du système et, finalement, que tous puissent trouver des bénéfices dans les correctifs apportés.

- Cinquième principe : envisager la recherche évaluative comme une activité permanente.

Une fois les conditions précédentes réalisées, il faut présumer qu'il existe une volonté commune d'évaluation, chez les participants, même si les motivations de départ ne sont pas forcément les mêmes. La permanence de l'évaluation suppose dès lors la mise en place de mécanismes autorisant d'effectuer des bilans à chacune des étapes dans l'implantation et le déroulement d'une action. Inscrite donc au programme comme activité régulière et continue, elle est susceptible, du même coup, d'être perçue comme moins menaçante. En pratique, il y aurait lieu de créer une unité d'évaluation au sein de l'équipe.

— Sixième principe : concevoir la recherche évaluative comme activité générée de l'intérieur.

L'entreprise d'évaluation, lorsqu'elle mobilise une volonté collective et des énergies chez les intéressés, s'impose comme initiative interne. En relevant le défi d'exercer un rôle dédoublé, bref de se soumettre à la critique des pairs et de procéder en même temps à son autocritique, le participant ne renonce pas pour autant à l'aide d'une expertise externe (auto-évaluation assistée) (Easton, 1984)

Au terme de cette introspection dans le champ de la recherche évaluative, un constat se dégage : celui de la richesse des expérimentations et de la maturité des questionnements. Certes, les impressions et réflexions que laissera au lecteur cette publication n'épuisent pas le thème complexe du rapport entre la recherche évaluative, la pratique sociale et la décision politique. Mais, le moins qu'on puisse admettre, c'est qu'elle éclaire sur des pistes d'orientation possibles, suggère des virages à négocier et, surtout, par la vigilance qu'elle inspire, nous prévient d'un double piège : celui d'une dépendance trop étroite du 
chercheur envers les institutions socio-politiques, et celui d'une distanciation trop prononcée du scientifique vis-à-vis les nouvelles réalités de l'intervention.

\section{Notes}

1 Cet article a été conçu en équipe autour de trois thèmes ou sections distinctes, qui ont par la suite été rédigées par des personnes différentes. Toutefois, les auteurs en acceptent la responsabilité collective.

2 L'article de Ricardo Zúñiga voulait, par ailleurs, poser le débat épistémologique entourant ce type de recherche.

3 Régis Blais a traité avec grand intérêt du problème des résistances à l'évaluation en milieu institutionnel.

4. À cet égard, il peut être intéressant de revoir les articles de Jacques Plante à propos d'un modèle d'évaluation institutionnelle, et celui de Francine Ouellet qui traite des aléas d'une pratique d'évaluation de programme.

5 À ce propos, l'article d'André Beaudoin, dans le présent numéro, démontre comment le single case design peut constituer un outil d'analyse d'une pratique dans un contexte d'évaluation formative. L'article de Lise DarveauFournier et d'Alice Home s'inscrit également dans cette optique, de même que l'expérience d'évaluation d'un modèle d'intervention féministe présentée par Maryse Rinfret-Raynor, Michèle Larouche et Ann PâquetDeehy.

6 On consultera, à ce sujet, l'article de Laurent Chambaud, Robert Mayer et Gabrielle Richard qui, dressant un bilan de la recherche-action menée dans certains milieux de pratique, suggère l'idée que la démarche d'évaluation se profile derrière un type particulier de recherche-action.

7 La démarche d'évaluation de Marie Bruneau, Michel Laflamme et MarieJosée Linteau est, à cet égard, assez révélatrice.

\section{Références bibliographiques}

ALKIN, M.C., "Evaluation theory development", Evaluation Comment, vol. 2, no 1, 1969: 1-7.

AlLAL, L., "Stratégies d'évaluation formative : conceptions psychopédagogiques et modalités d'application", dans: L. Allal, J. Cardinet et Ph. Perrenoud (éds), L'évaluation formative dans un enseignement différencié, Berne, Peter Lang, 1979.

ALLAL, L., Evaluation formation : entre l'intuition et l'instrumentation, Neuchâtel, Institut romand de recherches et de documentation pédagogiques, 1981. ("Cahier du GCR ", 1.) 
Beaudoin, A., Après 65 ans, Sherbrooke, Conseil régional de bien-être de Sherbrooke, 1972, 2 volumes.

Bloom, M. et J. FisCher, Evaluation Practice. Guidelines for the Accountable Professional, Englewood Cliffs (N.J.), Prentice-Hall, 1982.

CAMPBell, D.T. et J.C. StAnley, Experimental and Quasi-Experimental Designs for Research, Shokie (III.), Rand McNally, 1966.

CARDINET, J., "Des instruments d'évaluation pour chaque fonction", Mesure et évaluation en éducation, vol. 8, nos 1 et 2, 1985: 45-118.

CARO, F.G. (éd.), Readings in Evaluation Research, New York, Russell Sage, 1971.

CoOK, T.D. et C.S. REICHARDT (éds), Qualitative and Quantitative Methods in Evaluation Research, Beverly Hills (Cal.), Sage, 1979.

Crane, J.A., "The Power of social intervention experiments to discriminate differences between experimental and control groups", Social Service Review, vol. $50, n^{\circ} 2,1976$ : 224-242.

EAston, P., L'éducation des adultes en Afrique noire. (Manuel d'auto-évaluation assistée), tomes 1 et 2, Paris, Karthala, 1984.

Edwards, W., "Social utilities", The Engineering Economist, 1977: 119-129. ("Summer Symposium Series", 6.)

Edwards, W., M. GuttentaG et K. SNAPPER, "A decision-theoretic approach to Evaluation Research", dans : E.L. STRUENING et M. GUTTENTAG, Handbook of Evaluation Research, Beverly Hills (Cal.), Sage, 1975 : 139-182.

Freeman, H.E., "The present status of evaluation research", dans: M. GuttentaG et S. SAAR (éds), Evaluation Studies Review Annual, Vol. 2, Beverly Hills (Cal.), Sage, 1977 : 17-52.

GaUthier, B., "La recherche-action ", dans : B. GauthieR (dir.), Recherche sociale, de la problématique à la collecte des données, Sillery, Les Presses de l'Université du Québec, 1984.

GAUTHIER, Benoît, Méta-évaluation en Affaires sociales: analyse de cent cas de recherches évaluatives, Hull, Conseil québécois de la recherche sociale, 1982.

GuBA, E.G. et Y.S. Lincoln, Effective Evaluation, San Francisco, Jossey-Bass, 1983.

Hammond, R.L., tiré de StufflebeAM, Daniel I. et al., L'évaluation en éducation et la prise de décision, Victoriaville, N.H.P, 1980 : p. 278.

Hudson, W.H., The Clinical Measurement Package. A Field Manual, Homewood (III.), Dorsey Press, 1982.

Kaufman, R.A., Educational System Planning, Englewood Cliffs (N.J.), PrenticeHall, 1972.

KIRESUK, T.J. et G. GARWICK, "Basic goal attainment scaling procedures", dans: B.R. Compton et B. Gallaway, Social Work Processes, Homewood (III.), Dorsey Press, 1979 : 412-420.

LECOMTE, R. et L. RUTMAN, Introduction aux méthodes de recherche évaluative, Québec, Les Presses de l'Université Laval, 1982.

LECOMTE, R., "Les paradigmes méthodologiques en recherche évaluative : leurs fondements et leurs répercussions", dans: R. LECOMTE et L. RUTMAN, Introduction aux méthodes de recherche évaluative, Québec, Les Presses de l'Université Laval, 1982: 1-22. 
LefrançoIS, R. et M.H. SOULet, Le système de la recherche sociale, Tome I: La recherche sociale dans l'État, Sherbrooke, Département de service social, 1983. ("Recherches sociales".)

MARTIN, J.-M., Rapport de la Commission d'enquête sur le logement de la Cité de Québec, vol. I (1961), vol. II et III (1962), vol. IV (1963).

Mastow, A.H., Vers une psychologie de l'être, Paris, Fayard, 1972.

Mayer, R. et E. Greenwood, The Design of Social Policy Research, Englewood Cliffs (N.J.), Prentice-Hall, 1980.

Metfessel, M.S. et W.B. MiChAEL, "Paradigm involving multiple criterion measures for the evaluation of the effectiveness of school programs", Education and Psychological Measurement, vol. 27, n 4, 1967 : 931-943.

MUCHSLER, E., "Using single-case evaluation procedures in a family and children's agency : integration of practice and research ", Journal of Social Service Research, vol. 3, no 1, 1979: 115-134.

O'BRIEN, D.W., "Integrating clinical practice and research: the promise and design of single subject design methodology ", exposé présenté au Congrès des Sociétés Savantes, Ottawa, juin 1985.

Patton, M.Q., Qualitative Evaluation Methods, Beverly Hills (Cal.), Sage, 1980.

PATtON, M.Q., Utilization-Focused Evaluation, Londres, Sage, 1978 ; cité dans : D. TARSCHY, op. cit., p. 376.

Plante, J., "La notion de besoin et l'évaluation des programmes", Au fil des événements, Québec, Université Laval, 10 novembre 1983, p. 11.

Posavac, E.J. et R.J. Carey, Program Evaluation Methods and Case Studies, (2e édition), Englewood Cliffs (N.J.), Prentice-Hall, 1985.

Provus, M., "Presentation of the model", dans: Discrepancy Evaluation for Educational Program Improvement and Assessment, Berkeley (Calif.), McCutchan, 1971: 45-49.

RAYNOR, P., "L'évaluation avec un œil fermé: l'approche empirique dans la recherche en travail social ", Les Cahiers de la recherche sur le travail social, no 10, 1986 : 79-94.

RENARD, R., "Recherche scientifique et aide à la décision, gestion administrative et gestion rationnelle», Recherches sociologiques, vol. 16, no 1, 1985 : 143-169.

ROSENBLATt, A., "The practitioner's use and evaluation of research ", Social Work, vol. 13, no 1: 53-59.

RoskIN, M., "Evaluation research : bridge between social work, administrator, practitioner and researcher", International Social Work, vol. 22, no 1, 1979: 27-32.

Rossı, P.H., Evaluating Social Programs: Theory, Practice and Politics, Edited by Peter H. Rossi and Walter Williams, New York, Seminar Press, 1972.

Rossi, P.H. et H.E. Freeman, Evaluation a Systematic Approach, (3e édition), Beverly Hills (Cal.), Sage, 1985.

Rossı, P.H. et W. Willıams, Evaluating Social Programs, New York, Seminar Press, 1972. 
RUTMAN, L., "Recherche formative et évaluabilité de programme», dans: R. LeCOMTE et L. RutMan (éds), Introduction aux méthodes de recherche évaluative, Québec, Les Presses de l'Université Laval, 1982 : 65-78.

Sanchez, J., "L'évaluation entre la recherche et l'action", Propos sur la rechercche médico-sociale, Paris, CTNERHI, 1984.

SeChrest, L., S.G. West, M.A. Philips, R. Redner, et W. Yeaton, "Introduction", Evaluation Studies Review Annual, vol. 4, L. Sechrest et al. (éds), Beverly Hills (Calif.), Sage, 1979: 15-39.

SHAW, I. et R. WALTON, «Education for practice : former students' attitudes to a social work course", Contemporary Social Work Education, vol. 2, no 1, 1978: 15-29.

STAKE, R.E., "The countenance of educational evaluation", Teacher's College Record, 68, no 2, 1967 : 523-540.

SAINT-Arnaud, Y., La personne humaine, Montréal, Éditions du CIM/Éditions de I'Homme, 1974.

Stufflebeam, D.L. et al., L'évaluation en éducation et la prise de décision, Victoriaville, NHP, 1980.

SuCHMAN, E.A., Evaluative Research, New York, Russell Sage Foundation, 1967.

TARSCHYS, D., "Fluctuations in the political demand for policy analysis ", dans : S.E. SPIRO et E. YUCHTMAN-YAAR, Evaluating the Welfare State (Social and Political Perspectives), New York, Academic Press, 1983 : 375-385.

Tyler, R.W., Basic Principles of Curriculum and Instruction, Chicago (Ill.), University of Chicago, 1950.

Tyler, R., R. Gagné et M. SCRIven, Perspectives of Curriculum Evaluation, Chicago, University of Chicago Press, 1967.

VON SCHOENBERG, B., Les points de vue des clients et des citoyens : leur place dans l'évaluation de programme, Québec, Ministère de la santé et des services sociaux, Direction de l'évaluation des programmes, Québec, 1985.

WEINBERG, H., "Using policy analysis in congressional budgeting", dans: F.M. ZWEIG (éd.), Evaluation in Legislation, London, Sage, 1979: 28-44.

WeISS, C.H., "Policy evaluation as societal learning", dans: S.E. SPIRO et E. Yuchtman-YaAR, Evaluating the Welfare State (Social and Political Perspectives), New York, Academis Press, 1983: 361-375.

WeIsS, C.H., Evaluation Research, Englewood Cliffs (N.J.), Prentice-Hall, 1972.

WHOLEY, J.S., "Using evaluation to increase program performance", Evaluation Studies Review Annual, vol. 6, éd. H.E. Freeman \& M.A. Solomon, Beverly Hills, (Cal.), Sage, 1981 : 55-70.

ZIMBALIST, S., "The single-case research in developmental perspective: mainstream or tangent?", Journal of Education for Social Work, vol. 19, no 1, $1983: 6-66$. 